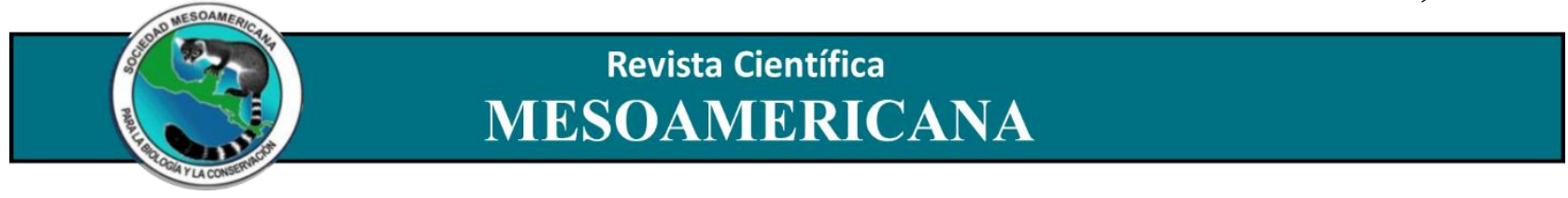

\title{
Descubrimiento de alcaloides en la piel de la rana Pristimantis gaigei, indicios de mimetismo Mülleriano en dos poblaciones de Panamá
}

\author{
Karol M. Gutiérrez-Pineda ${ }^{1,4^{*}}$ iD , Roger Morales ${ }^{2} \&$ Abel Batista $^{3,5}$ \\ 1Escuela de Química - Facultad de Ciencias Naturales y Exactas - Universidad Autónoma de Chiriquí. \\ ${ }^{2}$ Escuela de Biología - Facultad de Ciencias Naturales y Exactas - Universidad Autónoma de Chiriquí. \\ ${ }^{3}$ Vicerrectoría de Investigación y Postgrado - Universidad Autónoma de Chiriquí. \\ 4Fundación Pro-Conservación de los Primates Panameños, Ciudad de Panamá, Panamá. \\ ${ }^{5}$ Museo de Historia Natural, Universidad Autónoma de Chiriquí. \\ *Contacto: gutierrezk@fcprimatespanama.org
}

Fecha de Recepción: 28-VIII-2019. Fecha de Aceptación: 23-V-2020. Fecha de Publicación: 25-VI-2020.

\section{RESUMEN}

La rana Pristimantis gaigei ha sido utilizada como modelo de mimetismo batesiano, adoptando el aposematismo (coloración llamativa) de su modelo, la rana venenosa Phyllobates lugubris. Los estudios sobre la composición química de su piel son escasos. Considerando que se han reportado alcaloides en otras especies de ranas consideradas miméticas y sin toxinas por mucho tiempo, queremos corroborar la presencia o ausencia de algún tipo de alcaloide en el extracto de piel de dos poblaciones de P. gaigei (PJIC001-Parque Nacional Altos de Campana y BJIC002-Parque Internacional La Amistad-Atlántico) en Panamá. Para la caracterización fitoquímica de los alcaloides se utilizó las pruebas de Dragendorff, vapores de yodo y radiación de luz ultravioleta (365nm). Igualmente se empleó la técnica de cromatografía de capa fina (CCF), con dos fases móviles diferentes (cloroformo/metanol 9:1 y acetonitrilo/agua 9:1) y poder identificar el perfil químico de los compuestos. Tanto las muestras PJI001 Y BJI002, dieron positivas para la prueba de Dragendorff. Con capa fina se logró separar y caracterizar la presencia de más de un compuesto de alcaloide. Existió diferencia en la composición y comportamiento químico entre los dos extractos, donde la muestra de PJIC001 presenta compuestos entre una polaridad media y muy polares, con una composición química más abundante, para la muestra BJIC002, presentó compuestos con alta polaridad y baja polaridad. Estos resultados demuestran la presencia de diferentes compuestos de alcaloide en la piel de la P. gaigei, siendo un indicio de un mimetismo mülleriano, logrando adquirir un posible modelo químico para su defensa.

Palabras Clave: alcaloides, anfibios, defensa química, ecología química, mimetismo.

\section{Discovery of alkaloids in the skin of the frog Pristimantis gaigei, indications of Müllerian mimicry in two populations of Panama}

\section{ABSTRACT}

The frog Pristimantis gaigei has been used as a model of Batesian mimicry, adopting the aposematism (striking coloration) of its model, the poisoned frog Phyllobates lugubris. Studies on the chemical composition of its skin are scarce. Considering that alkaloids have been reported in other frog species 
considered mimetic and without toxins for a long time, we want to corroborate the presence or absence of some type of alkaloid in the skin extract of two populations of P. gaigei (PJIC001-Altos de Parque Nacional de Altos de Campana and BJIC002-La Amistad-Atlántico International Park) in Panama. For the phytochemical characterization of the alkaloids, Dragendorff tests, iodine vapors and ultraviolet light radiation (365nm) were used. The technique of thin layer chromatography (TLC) was also used, with two different mobile phases (chloroform / methanol 9: 1 and acetonitrile / water 9: 1) and to identify the chemical profile of the com-pounds. Both samples PJI001 and BJI002, tested positive for the Dragendorff test. With thin layer it was possible to separate and characterize the presence of more than one alkaloid compound. There was a difference in the composition and chemical behavior between the two extracts, where the sample of PJIC001 presents compounds between a medium and very polar polarity, with a more abundant chemical composition, for the sample BJIC002, it presented compounds with high polarity and low polarity. These results demonstrate the presence of different alkaloid compounds in the skin of $P$. gaigei, being an indication of a müllerian mimicry, managing to acquire a possible chemical model for its defense.

Keywords: defense, chemical ecology, amphibians, alkaloids, mimicry.

\section{INTRODUCCIÓN}

En el grupo de los anuros encontramos especies que hacen diferentes tipos de mimetismo (Symula et al., 2001; Sherratt, 2008; Browm et al., 2011; Twomey et al., 2013) las cuales han tenido la capacidad de aprender y trasmitir información fenotípica y química, como un mecanismo de defensa ante una alta depredación (Stuckert et al., 2014). Existen dos tipos de mimetismo a partir de la función básica de las especies, el mimetismo mülleriano (defensa química-toxicidad) y mimetismo batesiano (defensa aposemática) (Margalef, 1977; Díaz, 2011).

El aposematismo en anuros está relacionado con la necesidad del espécimen por defenderse contra depredadores, adquiriendo reflejos que lo hacen ver como no atacable, con una coloración advertidora (Margalef, 1977; Díaz, 2011). De esta forma, el depredador tiende a modificar su dieta, relacionando los colores llamativos con sabor desagradable (Ruxton et al., 2004; Díaz, 2011). Mientras que la función que cumple el modelo de defensa química es principalmente de contrarrestar microorganismos con moléculas biaoactivas y en potenciales depredadores, que va desde sabores desagradables hasta ser letal (Saporito et al, 2004; Daly et al., 2005; Gutiérrez et al., 2018).

Estas moléculas bioactivas han sido identificadas como aminas biogénicas, péptidos, proteínas, bufadienólidos, tetrodotoxinas y alcaloides lipofílicos (Daly et al., 2005; Weldon, 2006; Saporito et al., 2009; et al., 2012; Gutiérrez et al., 2018). En la familia Dendrobatidae, los alcaloides son las más importantes y juegan un papel crucial en la defensa contra depredadores (Margalef, 1977; Daly et al., 2005; Bayazit, 2010; Saporito et al., 2012).

En la actualidad la unión de estas dos características es consideradas como una innovación evolutiva, que implica rasgos como coloración, desarrollo corporal, comportamiento y dieta especializada, que van de la mano con la toxicidad, y sabor desagradable (Tullberg et al., 1996; Díaz, 2011), esto les permite obtener mayor cantidad de recursos que anteriormente no tenían al alcance (Lindquist et al., 1996; Speed et al., 2010).

La especie Pristimantis gaigei presenta variación de colores a través de su distribución (Lynch, 1985). Las poblaciones que se encuentran en Colombia presentan coloraciones parduscas (no miméticas), 
mientras que las ranas de poblaciones de América Central son negras con bandas dorso-laterales amarillas o anaranjadas en un fondo negro que las hace resaltar (Lynch, 1985; Leenders, 2016). Estas características fenotípicas hacen que se consideren como un caso de mimetismo batesiano, asemejando rasgos llamativos de una especie venenosa, la Phyllobates lugubris. Gracias a este tipo de mimetismo la especie logra ganar protección contra depredadores potenciales, al aparentar una especie peligrosamente tóxica (Leenders, 2001; Bayazit, 2010, Leenders, 2016). Alertando su peligrosidad y evitando su depredación, pero no poseen el modelo de defensa química, siendo realmente inofensivas y comestibles (Bayazit, 2010).

La distribución de la P. gaigei va desde el Sudoeste de Costa Rica, Panamá y Oeste de Colombia (Lynch, 1985). Este estudio abarca dos áreas de colecta en diferentes provincias de Panamá; Bocas del Toro, parte del nordeste de Panamá (Caribe) y Panamá Oeste (Pacífico). La especie P. gaigei, ha sido descrita como una especie que realiza mimetismo batesiano (Lynch, 1985; Leenders, 2016), sin defensa química-alcaloides, (Myers et al., 1983), donde solo imita el aposematismo de P. lugubris (Figura 4). Se conocen muy pocos casos de mimetismo mülleriano en anuros (Sherratt, 2008; Bayazit, 2010; Browm et al., 2011; Twomey et al., 2013), por ejemplo, el caso de la rana Ranitomeya imitator, la cual es considerada como un ejemplo para mimetismo mülleriano (Symula et al., 2001; Stuckert et al., 2014). El objetivo de esta investigación fue comprobar la presencia de alcaloides, en la piel de dos poblaciones de P. gaigei, para reforzar la hipótesis de mimetismo propuesta para esta especie.

\section{MATERIALES Y MÉTODOS}

\section{Área de estudio}

Bajo el permiso de colecta SE/A-33-18, dado por el Ministerio de Ambiente, se tomó un espécimen del Parque Internacional La Amistad, Provincia de Bocas del Toro, en el valle de Yorkín, cabeceras del río Tskui, antiguo bosque secundario en una pendiente pronunciada de borde menor con muchas palmeras (09²7'56"N, 8251'52”O), a 435 msnm, presenta un Bosque Húmedo Tropical de tierras bajas (Holdridge, 1967), y un espécimen del Parque Nacional Altos de Campana, Provincia de Panamá Oeste, en el sendero rana dorada, orillas del nacimiento del río Capira, conjunto de pequeñas cuevas, con poca vegetación alrededor $\left(08^{\circ} 43^{\prime 2} 28^{\prime \prime} \mathrm{N}, 79^{\circ} 56^{\prime} 56^{\prime \prime}\right.$ ) ), a $860 \mathrm{msnm}$, presenta un Bosque Muy Húmedo Premontano.

\section{Colecta y Preparación de los especímenes}

Se realizó giras nocturnas exploratorias en los diferentes sitios de muestreos con una lámpara de cabeza. Luego de ubicar los especímenes, se colectó uno en cada sitio y fueron colocadas en bolsas plástica con aire, agua y material vegetal conservando la humedad, para posteriormente ser llevado al laboratorio (Erazo et al., 2016). Los especímenes fueron sacrificados mediante el proceso de congelación, evitando la contaminación de la piel con agentes químicos. Posteriormente la misma fue retirada mediante disección, la muestra fue colocada en un vial con 1-2mL de etanol al 90\%, durante una semana para extraer los alcaloides (Daly, 1994). A cada extracto se le colocó el código del área de colecta respectivo. Parque Nacional Alto Campana en Panamá Oeste PJIC001 y Extracto del Parque Internacional la Amistad en Bocas del Toro BJIC002.

Caracterización química (Identificación de alcaloides) - Técnica de Cromatografía de Capa Fina (CCF) Se utilizó un tubo capilar $(0.5 \mathrm{~mm}$ de diametro por $10 \mathrm{~cm}$ de longitud) para colocar una pequeña muestra del extracto etanólico en una placa de sílica (TLC Silica gel 60 F254), y luego se agregó el reactivo de Dragendorff, la formación de precipitados y cambios de color indican la presencia o ausencia de alcaloides en la muestra (Hostettmann et al., 2008). Se realizó el desarrollo de la placa con los diferentes extractos de la piel (PJIC001 y BJIC002), se utilizó diferentes fases móviles, entre 
ellas, (cloroformo:metanol 9:1), (acetonitrilo:agua 9:1), para determinar el comportamiento químico de los compuestos presentes.

Después del secado, la placa se observó en Luz Ultravioleta (UV-365nm) y se calculó los Rf (relación de frentes) de las manchas, de igual forma se revelaron con el reactivo de Dragendorff y cámara de yodo. La visualización de manchas marrones de color chocolate sobre el fondo blanco de la placa, se considera prueba positiva para alcaloides. Luego de esto se tomaron fotografías digitales de todas las placas de CCF, ya que el reactivo de Dragendorff y el vapor de yodo producen un registro no permanente. Cada carril sobre la placa de CCF se colocó como positivo, negativo, o de rastreo para alcaloides. Un resultado positivo se registró cuando aparecieron manchas oscuras, mediante la exposición al vapor de yodo; un resultado negativo se registró cuando nada aparecía (Hostettmann et al., 2008). Es importante resaltar que los extractos individuales de diversas especies se compararon lado a lado.

\section{RESULTADOS}

Los extractos de piel de P. gaigei (PJIC001 y BJIC002) reaccionaron ante la presencia del reactivo Dragendorff, con un precipitado de color chocolate oscuro, evidenciando la presencia de alcaloides (Figura 1). Los datos obtenidos en la cromatografía de capa fina muestran que los extractos presentan diferencias en la composición y comportamiento químico (Figura 2, Figura 3). En la fase móvil no polar (cloroformo:metanol 9:1) se logró la separación de tres Rf en ambos extractos PJIC001 y BJIC002. En la fase móvil polar (acetonitrilo:agua 9:1), para PJIC001 separó cinco Rf y BJIC002 tres Rf. Cada uno de los puntos separados por las fases móviles re-sultaron positivas para las tres pruebas de caracterización fitoquímica de alcaloides (Cuadro 1).

\begin{tabular}{|c|c|c|}
\hline $\begin{array}{c}\text { Código de las muestras } \\
\text { Reactivo Dragendorff } \\
\text { (Control) }\end{array}$ & PJIC001 & BJIC002 \\
\hline $\begin{array}{c}\text { Muestra } \\
\text { Muestra + Reactivo } \\
\text { Dragendorff }\end{array}$ & & \\
\hline
\end{tabular}

Figura 1. Caracterización de alcaloides con el reactivo Dragendorff. La formación del precipitado chocolate es indicativo de positivo para alcaloides en ambos extractos de las muestras (PJIC001 y BJIC002).

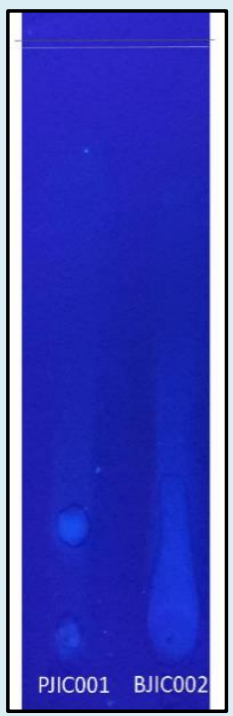

Figura 2. Representación de la separación de alcaloides en los extractos (PJIC001 y BJIC002) con la Técnica de Cromatografía de Capa Fina (TLC) y revelados con Luz Ultravioleta (365nm).
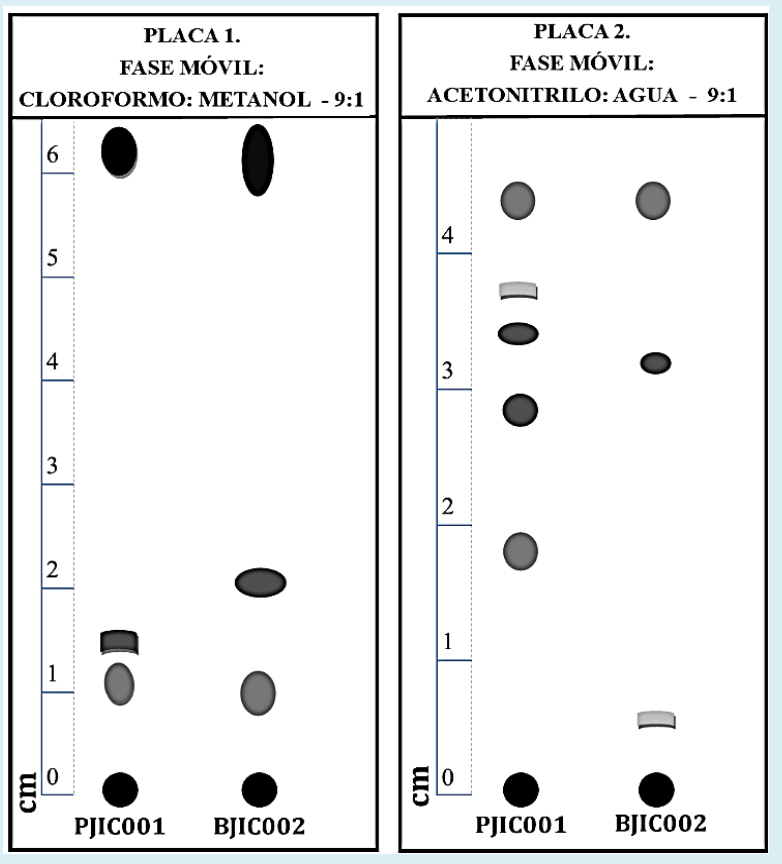

Figura 3. Modelo de separación de alcaloides en los extractos. Placa 1, Fase móvil no polar. Placa 2. Fase móvil polar. 
Cuadro 1. Datos obtenidos en la caracterización química de alcaloides y en el perfil químico de los extractos de piel de la Pristimantis gaigei.

\begin{tabular}{|c|c|c|c|c|c|c|}
\hline Fase móvil - & \multicolumn{4}{|c|}{ Cloroformo: Metanol - Proporciones 9:1 } & \multicolumn{2}{|c|}{ - CCF Fase normal } \\
\hline Nombre & $\begin{array}{l}\text { Recorrido de la } \\
\text { fase móvil }(\mathrm{cm})\end{array}$ & $\begin{array}{l}\text { Recorrido del } \\
\text { soluto }(\mathrm{cm})\end{array}$ & $\mathrm{Rf}$ & $\begin{array}{l}\text { Prueba de } \\
\text { Dragendorff }\end{array}$ & $\begin{array}{c}\text { Prueba con } \\
\text { Yodo }\end{array}$ & $\begin{array}{c}\text { Cámara UV } \\
\text { 365nm }\end{array}$ \\
\hline \multirow{4}{*}{$\begin{array}{l}\text { Muestra } \\
\text { PJIC001 }\end{array}$} & 6.5 & 1 & 0.15 & +++ & +++ & azul \\
\hline & 6.5 & 1.3 & 0.2 & +++ & +++ & azul \\
\hline & 6.5 & 6.3 & 0.97 & +++ & +++ & azul \\
\hline & 6.5 & 0 & 0 & +++ & +++ & azul \\
\hline \multirow{4}{*}{$\begin{array}{l}\text { Muestra } \\
\text { BJIC002 }\end{array}$} & 6.5 & 0.9 & $\begin{array}{c}0.13 \\
8\end{array}$ & +++ & +++ & azul \\
\hline & 6.5 & 2 & 0.3 & +++ & +++ & azul \\
\hline & 6.5 & 6.3 & 0.97 & +++ & +++ & azul \\
\hline & 6.5 & 0 & 0 & +++ & +++ & azul \\
\hline \multicolumn{3}{|c|}{ Fase móvil - } & \multicolumn{2}{|c|}{ - Proporciones 9:1 } & \multicolumn{2}{|c|}{ - CCF Fase reversa } \\
\hline \multirow{6}{*}{$\begin{array}{l}\text { Muestra } \\
\text { PJIC001 }\end{array}$} & 5 & 1.7 & 0.34 & +++ & +++ & azul \\
\hline & 5 & 2.8 & 0.56 & +++ & +++ & azul \\
\hline & 5 & 3.4 & 0.68 & +++ & +++ & azul \\
\hline & 5 & 3.8 & 0.76 & +++ & +++ & azul \\
\hline & 5 & 4.3 & 0.86 & +++ & +++ & azul \\
\hline & 5 & 0 & 0 & +++ & +++ & azul \\
\hline \multirow{4}{*}{$\begin{array}{l}\text { Muestra } \\
\text { BJIC002 }\end{array}$} & 5 & 0.4 & 0.1 & +++ & +++ & azul \\
\hline & 5 & 3.2 & 0.64 & +++ & +++ & azul \\
\hline & 5 & 4.3 & 0.86 & +++ & +++ & azul \\
\hline & 5 & 0 & 0 & +++ & +++ & azul \\
\hline
\end{tabular}

Leyenda: Extracto del espécimen del Parque Nacional Alto Campana (PJIC001); extracto del espécimen del Parque Internacional La Amistad-Atlántico (BJIC002), Panamá. Pruebas con el reactivo Dragendorff y la cámara de yodo: negativo (--); positivo (+++); Prueba con el UV: positivo (azul), negativo (sin color).

\section{DISCUSIÓN}

De acuerdo con los métodos de caracterización de alcaloides utilizados por Myers y Daly (1976) y replicados en este estudio, se demuestra la presencia de alcaloides en los extractos de piel de P.gaigei en dos sitios de Panamá. Anteriormente especies de la familia Craugastoridae habían sido evaluadas en busca de alcaloides y péptidos dando como resultado la ausencia de ellos (König et al., 2015). En este trabajo $P$. gaigei evidencia la primera especie de la familia Craugastoridae que presenta alcaloides en la piel.

Con la técnica de CCF logramos determinar que el perfil y comportamiento químico de los compuestos presentes en ambos extractos eran diferentes. Esta misma técnica la implementaron con la fase móvil Cloroformo:metanol (9:1) en los inicios de caracterización y separación de alcaloides en las ranas dardo venenosas (Dendrobatidae) (Myers \& Daly, 1976; Myers et al., 1978) y la tomamos como referencia para este estudio, logrando la separación de varios $R_{\mathrm{f}}$, similares a los encontrados 
previamente para dendrobatidos (Myers \& Daly, 1976; Myers et al., 1978). Para esta misma fase móvil, podemos sugerir la presencia de tres clases de alcaloide: pumilliotoxina, histrionicotoxina, decahidroquinolinas. En el caso de la muestra PJIC001, presento $\mathrm{R}_{\mathrm{f}}$ de 0.20 y 0.15 , los cuales corresponden a los $\mathrm{R}_{\mathrm{f}}$ de la pumiliotoxina $\mathrm{B}$ y histrionicotoxina 239, respectivamente. En la muestra BJIC002, se identificaron dos $\mathrm{R}_{\mathrm{f}}$, estos son 0.14 y 0.30 que corresponde de igual forma a lo reportado para los $\mathrm{R}_{\mathrm{f}}$ de la histrionicotoxina 239 y a la decahidroquinolina respectivamente.

No se puede asegurar que los valores de estos $R_{\mathrm{f}}$ son indicativos de estos alcaloides reportados previamente, es lo que se puede sugerir, ya que la cromatografía de capa fina no es suficiente para demostrar la identidad de un compuesto desconocido y tampoco podemos deducir que cada mancha contiene un solo alcaloide, pueden presentar más de uno (Myers et al., 1978). Queda por detallar y profundizar los estudios bioquímicos para identificar el o los alcaloides encontrados en nuestro análisis.

La segunda mezcla que utilizamos como fase móvil fue acetonitrilo: agua, en proporciones 9:1. Con esta fase logramos una mayor separación de compuestos para la muestra PJIC001, con la obtención de 5 señales. Para el caso de la muestra BJIC002, se tuvo una menor separación de la mezcla, con cuatro señales, las mismas fueron vistas en el UV, donde todas refractaron la luz, observándose una coloración azul, de igual forma todas reaccionaron con el reactivo Draguendorff, confirmando la presencia de alcaloides.

Lo que logramos obtener con esta fase móvil es que el perfil químico de los diferentes extractos presentan diferencias entre ellos, de la composición y en el comportamiento químico. Donde la muestra de PJIC001 presentó compuestos entre una polaridad media y muy polares, con una composición química más abundante. Mientras que para la muestra BJIC002, presentó compuestos de alta polaridad y baja polaridad. Esta diferencia química puede estar relacionada con la separación geográfica y el tipo de hábitat, en que se obtuvieron las dos muestras (Díaz, 2011).

Considerando que la pumiliotoxina ha sido reportada en la especie modelo P. lugubris (Daly et al., 2005) y es la molécula que se considera tiene una amplia distribución en todos los anuros que tengan algún tipo de defensa química (Saporito et al., 2004), podría estar presente igualmente en $P$. gaigei. Otros estudios aseguran que la dieta es uno de los factores más importantes en la adquisición de una defensa química (alcaloides). En el caso de la familia Dendrobatidae su defensa química proviene de una dieta secuestrada, basada en artrópodos (hormigas, milpiés, ácaros, escarabajos, etc.) (Daly et al., 1994; Daly et al., 1999; Saporito et al., 2009). Debido a que se ha encontrado alcaloides en grupos de ranas filogenéticamente distintos a la familia Dendrobatidae (Ranas dardo venenosas) (Rodríguez et al., 2011; Prates et al., 2012), debemos empezar a generar una base de datos sobre especies que en la actualidad se consideran que hacen mimetismo batesiano, como por ejemplo el espécimen de estudio P. gaigei, pero con pruebas sencillas como las utilizadas, podemos deducir si están logrando adquirir un modelo químico para su defensa. Al final, el éxito de una especie que hace mimetismo ya sea batesiano o mülleriano, es poder generar una señal de advertencia contra sus depredadores, 0 simplemente pasar desapercibida, logrando la supervivencia natural de su especie.

\section{CONCLUSIÓN}

Se logró la identificación de la presencia de alcaloides en los extractos de las diferentes poblaciones estudiadas de P. gaigei, por medio de tres pruebas de caracterización fitoquímica, Dragendorff, vapores de yodo y radiación de luz ultravioleta $(365 \mathrm{~nm})$, donde todas resultaron positivas. La cromatografía en capa fina se utilizó principalmente para saber el perfil químico preliminar de nuestras muestras. Donde se obtuvo diferencia entre los extractos, tanto en la composición química, 
como en el comportamiento fisicoquímico. La muestra de PJIC001 presenta compuestos entre polaridad media a muy polares, con una composición química más abundante. Mientras que para la muestra BJIC002, presenta compuestos con alta polaridad y con baja polaridad.

En este estudio damos indicios de que P. gaigei presenta un mimetismo mulleriano (defensa químicaalcaloides) en vez del mimetismo batesiano. Las implicaciones de nuestro hallazgo son importantes para futuros estudios a nivel evolutivo y de estrategias de defensa en estas ranas. Considerando que aún desconocemos las estructuras moleculares específicas de estos alcaloides, podrían estar guardando compuestos importantes para uso potencial en la farmacocinética.

\section{AGRADECIMIENTOS}

Agradecemos al Centro de Investigación de Productos Naturales y Biotecnología (CINAPBIOT) de la Universidad Autónoma de Chiriquí (UNACHI), por prestar las instalaciones para la caracterización química de nuestras muestras. De igual forma queremos agradecer al Lic. Marcos Ponce, al estudiante Eric Barrías, por la colaboración en la colecta de las muestras. A la Lic. Milagros Centeno, Laura Patiño, Ph.D. por su asistencia en el laboratorio. A Pedro G. Méndez-Carvajal, Ph.D., por el apoyo en la revisión del borrador de este artículo y sus consejos en el mismo.

\section{CONFLICTO DE INTERESES}

Los autores presentes declaran que no existe intereses de conflicto.

\section{REFERENCIAS CITADAS}

ARANG0, G. 2008. Alcaloides y compuestos nitrogenados, Facultad de Química farmacéutica, Universidad de Antioquia. Medellín, Colombia.

BAYAZIT, V. 2010. Biological activities of nanomaterials (bufadienolides, peptides and alkoloids) in the skin of amphibian on Gammarus pulex L. Digest Journal of Nanomaterials and Biostructures, 5(2): 347-354.

BROWN, J. L., TWOMEY, E., AMEZQUITA, A., DE SOUZA, M. B., CALDWELL, J. P., LOETTERS, S., ... \& PEPPER, M. 2011. A taxonomic revision of the Neotropical poison frog genus Ranitomeya (Amphibia: Dendrobatidae). Zootaxa, 3083(1): 1-120.

DALY, J. W., GARRAFFO, H. M., \& SPANDE, T. F. 1999. Alkaloids from amphibian skins. In: Pelletier, S. W. (ed). Alkaloids: chemical and biological perspectives. Pergamon New York, pp 1-161.

DALY, J. W., GUSOVSKY, F., MYERS, C.W., YOTSU-YAMASHITA, M. \& YASUMOTO, T. 1994. First occurrence of tetrodotoxin in a dendrobatid frog (Colostethus inguinalis), with further reports for the bufonid genus Atelopus. Toxicon, 32: 279-285

DALY, J. W., SECUNDA, S. I., GARRAFFO, H. M., SPANDE, T. F., WISNIESKI, A. \& COVER JR, J. F. 1994. An uptake system for dietary alkaloids in poison frogs (Dendrobatidae). Toxicon, 32(6): 657-663.

DALY, J. W., SPANDE, T. F. \& GARRAFFO, H. M. 2005. Alkaloids from amphibian skin: a tabulation of over eight-hundred compounds. Journal of natural products, 68(10): 1556-1575.

DíAZ, J. A. H. 2011. Beauty Does Not Always Denote Danger: Aposematic Syndrome in the Poison Frog Dendrobates auratus (Doctoral dissertation, Uniandes).

ERAZO LONDOÑo, J. P., RUANO MENESES, L. A. \& LÓPEZ PEÑA, A. 2016. Comparación entre dieta y composición de alcaloides de Dendrobates truncatus (Dendrobatidae) entre dos zonas con diferentes grados de perturbación en un bosque seco. Revista de Ciencias, 20(2): 95-107. 
GUTIÉRREZ, K., MORALES, R. \& PINO, J. 2018. Ranas dardo venenosas (Dendrobatidae) y su importancia en la bioprospección de moléculas bioactivas en los últimos tiempos: una revisión. Revista de Iniciación Científica, 4: 43-47.

HOSTETTMANN, K., GUPTA, M. P., MARSTON, A. \& FERREIRA, E. 2008. Manual de estrategias para el aislamiento de productos naturales bioactivos. Programa iberoamericano de Ciencia y Tecnología. CYTED.

KÖNIG, E., BININDA-EMONDS, O. R. \& SHAW, C. 2015. The diversity and evolution of anuran skin peptides. Peptides, 63: 96-117.

LEENDERS, T. 2001. A Guide to Amphibians and Reptiles of Costa Rica. Zona Tropical, Miami.

LEENDERS, T. 2016. A Field Guide to Amphibians of Costa Rica. Zona Tropical.

LINDQUIST, N. \& HAY, M. E. 1996. Palatabilidad y defensa química de las larvas de invertebrados marinos. Monografías ecológicas, 66(4): 431-450.

LYNCH, J. D. 1985. Mimetic and Non-Mimetic Populations of Eleutherodactylus gaigeae (Dunn) in lower Central America and Colombia (Amphibia: Anura, Leptodactylidae). Studies on neotropical fauna and environment, 20(4): 195-202.

MARGALEF, R. 1977. Comunicación y engaño. Aspectos e implicaciones de la crisis, advertencia y mimetismo, Revista de entomólogos de Ibéricos, 341-356

MYERS, C. W. \& DALY, J. W. 1976. Preliminary evaluation of skin toxins and vocalizations in taxonomic and evolutionary studies of poison-dart frogs (Dendrobatidae). Bull. Am. Mus. Nat. Hist, 157: 175-262.

MYERS, C. W. \& DALY, J. W. 1983. Dart-poison frogs. Scientific American, 248(2): 120-133.

MYERS, C. W., DALY, J. W. \& MALKIN, B. 1978. A dangerously toxic new frog (Phyllobates) used by Embera Indians of western Colombia, with discussion of blowgun fabrication and dart poisoning. Am Mus Novitates, 161: 307-366

PRATES, I., ANTONIAZZI, M. M., SCIANI, J. M., PIMENTA, D. C., TOLEDO, L. F., HADDAD, C. F. \& JARED, C. 2012. Skin glands, poison and mimicry in dendrobatid and leptodactylid amphibians. Journal of morphology, 273(3): 279-290.

RODRÍGUEZ, A., POTH, D., SCHULZ, S. \& VENCES, M. 2011. Discovery of skin alkaloids in a miniaturized eleutherodactylid frog from Cuba. Biol Lett, 7:414-418.

RUXTON, G., SHERRAT, T. \& SPEED, M. P. 2004. "The form and function of warning signals Avoiding Attack: The Evolutionary Ecology of crypsys, Warning Signals and Mimicry" New York: Oxford Univ. Press. 82-101 PP.

SAPORITO, R. A., DONNELlY, M. A., MADDEN, A. A., GARRAFFO, H. M. \& SPANDE, T. F. 2009. Sex-related differences in alkaloid defenses of the dendrobatid frog Oophaga pumilio from Cayo Nancy, Bocas del Toro, Panama. J Nat Prod, 73:317-321.

SAPORITO, R. A., DONNELLY, M. A., SPANDE, T. F. \& GARRAFFO, F. M. 2012. A review of chemical ecology in poison frogs. Chemoecology, 22:159-168. DOI 10.1007/s00049-011-0088-0.

SAPORITO, R. A., GARRAFFO, H. M., DONNELLY, M. A., EDWARDS, A. L., LONGINO, J. T. \& DALY, J. W. 2004. Formicine ants: an arthropod source for the pumiliotoxin alkaloids of dendrobatid poison frogs. Proceedings of the National Academy of Sciences, 101(21): 8045-8050.

SHERRATT, T. N. 2008. La evolución de la mímica mülleriana. Naturwissenschaften, 95: 681-695. 
SPEED, M. P, BROCKHURST, M. A. \& RUXTON, G. D. 2010. "The dual benefits of aposematism: predator avoidance and enhanced resource collection". Evolution, 64(6): 1622-1633.

STUCKERT, A. M., SAPORITO, R. A., VENEGAS, P. J. \& SUMMERS, K. 2014. Alkaloid defenses of co-mimics in a putative Müllerian mimetic radiation. BMC evolutionary biology, 14: 76. https://doi.org/10.1186/1471-214814-76

SYMULA, R., SCHULTE, R. \& SUMMERS, K. 2001. Molecular phylogenetic evidence for a mimetic radiation in Peruvian poison frogs supports a mullerian mimicry hypothesis. Proc R Soc Lond B, 268: 2405-2421.

TULLBERG, B. S. \& HUNTER, A. F. 1996. Evolution of larval gregariousness in relation to repellent defences and warning coloration in tree-feeding Macrolepidoptera: a phylogenetic analysis based on independent contrasts. Biological Journal of the Linnean Society, 57(3): 253-276.

TWOMEY, E., YEAGER, J., BROWN, J. L., MORALES, V., CUMMINGS, M. \& SUMMERS, K. 2013. Phenotypic and genetic divergence among poison frog populations in a mimetic radiation. PloS one, 8(2): e55443.

WELDON, P. J., KRAMER, M., GORDON, S., SPANDE, T. F. \& DALY, J. W. 2006. A common pumiliotoxin from poison frogs exhibits enantioselective toxicity against mosquitoes. Proceedings of the National Academy of Sciences, 103(47): 17818-17821.

Citar como: Gutiérrez-Pineda, K., Morales, R. \& Batista, A. 2020. Descubrimiento de alcaloides en la piel de la rana Pristimantis gaigei, indicios de mimetismo Mülleriano en dos poblaciones de Panamá. Mesoamericana 24(1): 49-57.

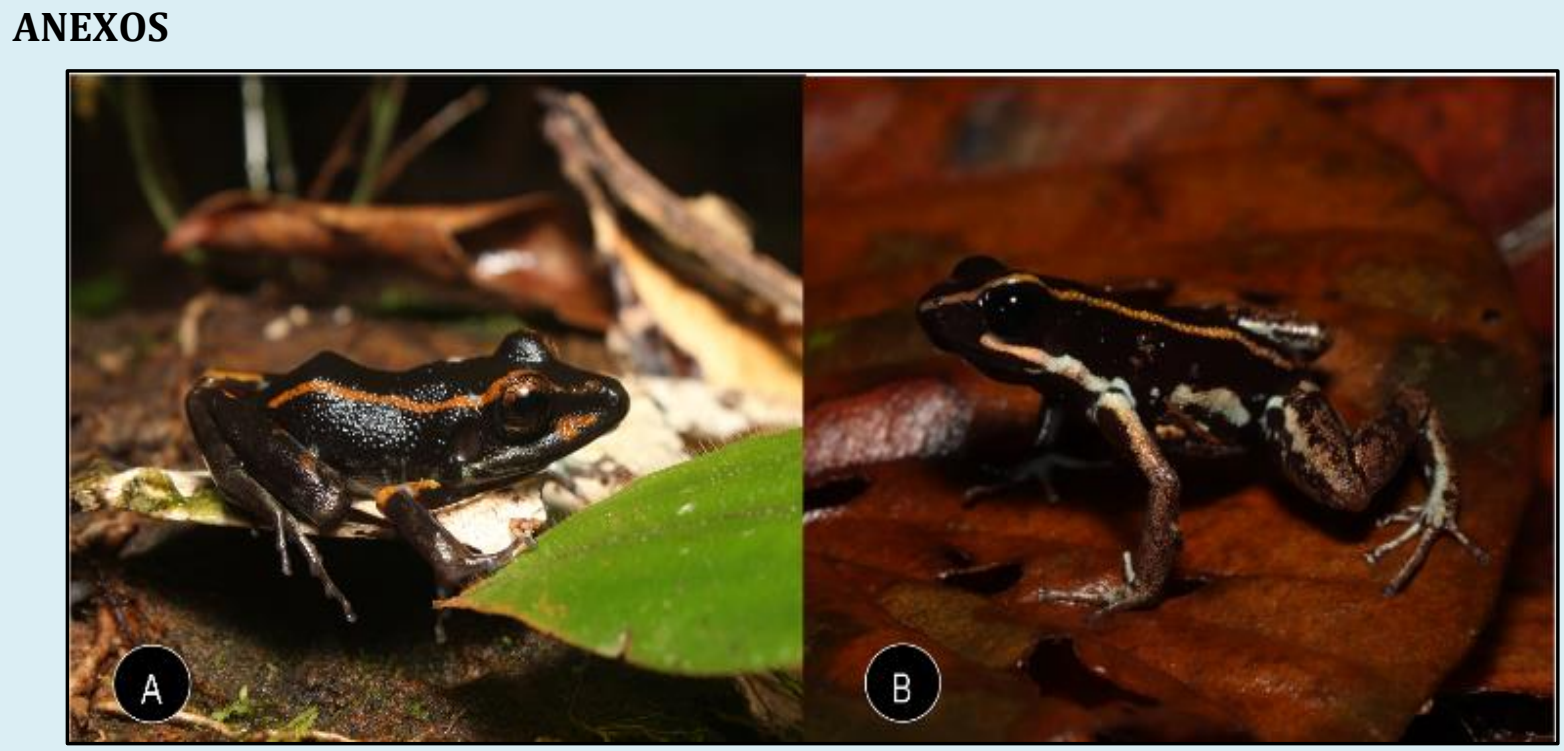

Figura 4. (A) Pristimantis gaigei, especie mimética; (B) Phyllobates lugubris, especie modelo 